Stud. Univ. Babeş-Bolyai Math. 63(2018), No. 4, 437-445

DOI: $10.24193 /$ subbmath.2018.4.02

\title{
Goldie absolute direct summand rings and modules
}

\author{
Truong Cong Quynh and Serap Şahinkaya
}

\begin{abstract}
In the present paper, we introduce and study Goldie ADS modules and rings, which subsume two generalizations of Goldie extending modules due to Akalan et al. [3] and ADS-modules due to Alahmadi et al. [7]. A module $M$ will be called a Goldie ADS module if for every decomposition $M=S \oplus T$ of $M$ and every complement $T^{\prime}$ of $S$, there exists a submodule $D$ of $M$ such that $T^{\prime} \beta D$ and $M=S \oplus D$. Various properties concerning direct sums of Goldie ADS modules are established.
\end{abstract}

Mathematics Subject Classification (2010): 16D40, 16E50, 16N20.

Keywords: Goldie extending modules, ADS modules, CS modules.

\section{Introduction}

The purpose of the present paper is to introduce and study Goldie ADS modules, which allow us to give a unified approach of Goldie extending modules and ADSmodules, introduced by E. Akalan et al. [3] and A. Alahmadi et al. [7], respectively. We define a Goldie ADS module by the property that for every decomposition $M=S \oplus T$ of $M$ and every complement $T^{\prime}$ of $S$, there exists a submodule $D$ of $M$ such that $T^{\prime} \beta D$ and $M=S \oplus D$. We study these modules, generalizing several results both on Goldie extending modules and ADS-modules. We show that a non-singular Goldie ADS module is an ADS module. We emphasize that our properties are of the same type as those for Goldie extending modules and ADS-modules, sharing similar limitations in studying certain properties, such as the closure of the respective class of modules under direct sums. We also analyze when a direct summand of Goldie ADS modules is a Goldie ADS and also when a direct sum of Goldie ADS module is Goldie ADS, by using the concepts of relative ejectivity. In the last section, we look at Goldie ADS property of some ring extensions. 


\section{Definitions and notions}

In this paper, $R$ will present an associative ring with identity and all modules over $R$ are unitary right modules. We also write $M_{R}$ to indicate that $M$ is a right $R$-module. We shall denote the fact that a submodule $N$ is essential in a module $M$ by $N \leq_{e} M$. The following generalization of relative injectivity is introduced in [3, Definition 2.1]. Let $N$ and $M$ be modules. $N$ is called $M$-ejective if, for each $K \leq M$ and each homomorphism $f: K \rightarrow N$, there exist a homomorphism $\bar{f}: M \rightarrow N$ and a $X \leq_{e} K$ such that $\bar{f}(x)=f(x)$, for all $x \in X$. M and $N$ is called mutually ejective if $M$ is $N$-ejective and $N$ is $M$-ejective. A submodule $K$ of $M$ is called fully invariant if $f(K) \subseteq K$ for every $f \in \operatorname{End}_{R}(M)$. Clearly 0 and $M$ are fully invariant submodules of $M$. The right $R$-module $M$ is called a duo module provided every submodule of $M$ is fully invariant. The singular submodule of a module $M$ will be denoted by $Z(M)=\left\{m \in M: m I=0\right.$ for some $\left.I \leq_{e} R_{R}\right\}$. A module $M$ is called singular (respectively non-singular) if $Z(M)=M$ (respectively $Z(M)=0$ ).

(CS): Every complement submodule of $M$ is a direct summand of $M$.

(C2): Every submodule of $M$ that is isomorphic to a direct summand of $M$ is itself a direct summand of $M$.

(C3): For any two direct summands $A$ and $B$ of $M$ with $A \cap B=0$, the sum $A+B$ is a direct summand of $M$.

A module $M$ is called is called continuous (respectively, quasi continuous) if $M$ satisfies (CS) and (C2) (respectively, (CS) and (C3)).

Let $M$ be an $R$-module and $X, Y \leq M$. In [3], the notion of $\beta$ relation on submodules $X, Y$ of $M$, denoted by $X \beta Y$, is defined such as $X \beta Y$ if and only if $X \cap A=0$ implies $Y \cap A=0$ and $Y \cap B=0$ implies $X \cap B=0$ for all $A, B \leq M$. A right module $M$ is Goldie extending if for each $X \leq M$, there exists a direct summand $D$ of $M$ such that $X \beta D . M$ is Goldie extending if and only if for each closed submodule $C$ of $M$ there is a direct summand $D$ of $M$ such that $C \beta D$.

Another notion generalizing extending property, ADS (Absolute Direct Summand) modules, was recently considered in [7]. It was introduced by Fuchs [10] for abelian groups and for general modules by Alahmadi, Jain and Leroy [7]. As the authors pointed out in [7], if $R$ is commutative then every cyclic $R$-module is ADS. Also every right quasi-continuous module is $\mathrm{ADS}$, but the converse is not true. However, a right ADS module which is also CS is quasi-continuous. Also in [14], Quynh and Koşan proved that every ADS module satisfies (C3). Hence, this is a class of modules between quasi-continuous modules and modules satisfying the (C3) condition. Quynh and Koşan gave also different characterizations of ADS modules and showed how to characterize semisimple modules and semisimple artinian rings using the ADS. The SC and SI rings were also characterized by the ADS notion in [14].

\section{Goldie absolute direct summand modules}

A module $M$ is called Goldie absolute direct summand (Goldie ADS) if, for every decomposition $M=S \oplus T$ of $M$ and every complement $T^{\prime}$ of $S$, there exists a submodule $D$ of $M$ such that $T^{\prime} \beta D$ and $M=S \oplus D$. 
A ring $R$ right Goldie ADS if the (right) $R$-module $R$ is Goldie ADS. We know that extending modules are Goldie extending, but need not be ADS. Similarly, Goldie extending modules do not necessarily satisfy Goldie ADS. Hence, the notions extending, Goldie extending, ADS and the property Goldie ADS are not directly related.

Example 3.1. Let $R=\mathbb{Z}_{2}\left[x_{1}, x_{2}, \ldots\right]$, where $x_{i}$ are commuting indeterminants satisfying the relations: $x_{i}^{3}=0$ for all $i, x_{i} x_{j}=0$ for all $i \neq j$, and $x_{i}^{2}=x_{j}^{2}$ for all $i$ and $j$. Then $R$ is a commutative, semiprimary ring with simple essential socle. But $R$ is not a self-injective ring (see [13, Example 5.45]). On the other hand, $R_{R}$ is soc- $R_{R}$-injective by [8, Example 5.7]. It follows that $R_{R}$ is soc- $\left(R_{R} \oplus R_{R}\right)$-injective by [8, Theorem 2.2(4)]. We have $\operatorname{Soc}\left(R_{R} \oplus R_{R}\right)=\operatorname{Soc}\left(R_{R}\right) \oplus \operatorname{Soc}\left(R_{R}\right)$ is finitely generated. Therefore $R_{R} \oplus R_{R}$ is soc- $\left(R_{R} \oplus R_{R}\right)$-injective by [8, Theorem 2.10]. Since $\operatorname{Soc}\left(R_{R} \oplus R_{R}\right)$ is essential in $R_{R} \oplus R_{R}$, then $R_{R} \oplus R_{R}$ is self-ejective by [3, Corollary 2.5(iii)]. It follows that for each decomposition $R_{R} \oplus R_{R}=A \oplus B, A$ and $B$ are mutually ejective by Lemma 3.11(2). It shows that $R_{R} \oplus R_{R}$ is Goldie ADS by Lemma 3.6. On the other hand, $R_{R} \oplus R_{R}$ is not $\mathrm{ADS}$. Indeed, if $R_{R} \oplus R_{R}$ is $\mathrm{ADS}$, then $R$ must be self-injective, a contradiction.

Example 3.2. Let $R$ be a triangle matrix ring over a field $K$. Then $R_{R}$ is CS. Note that $R_{R}$ is non-singular. Since $R_{R}$ is not a C3-module, $R_{R}$ is not ADS. It follows that $R_{R}$ is not Goldie ADS by Corollary 3.7.

Example 3.3 ([14, Example 2.10]). Let $K$ be a field and let $R=K[x, y] /\left\langle x^{2}, x y, y^{2}\right\rangle$. Assume that $S$ is any simple injective $R$-module. Let $M=R \oplus S$. Then $M$ is not a CS-module (since $R$ is indecomposable and not uniform). On the other hand, $R, S$ are relatively injective, and any two decompositions of $M$ are isomorphic (since $R$ and $\operatorname{End}(S)$ are local rings). Hence $M$ is an ADS module.

Let us mention the following equivalent conditions for Goldie ADS modules.

Lemma 3.4. The following conditions are equivalent for a module $M$.

1. $M$ is Goldie ADS.

2. For every decomposition $M=S \oplus T$ of $M$ and every complement $T^{\prime}$ of $S$, there exists a submodule $D$ of $M$ and $X$ of $M$ such that $X \leq_{e} T^{\prime}, X \leq_{e} D$ and $M=S \oplus D$.

Proof. (1) $\Rightarrow(2)$. Assume that $M$ is Goldie ADS and $M$ has a decomposition $M=$ $S \oplus T$. Let $T^{\prime}$ be a complement of $S$. Then there exists a submodule $D$ of $M$ such that $T^{\prime} \beta D$ and $M=S \oplus D$. Let $X=T^{\prime} \cap D$. Hence $X \leq_{e} T^{\prime}, X \leq_{e} D$.

$(2) \Rightarrow(1)$ is obvious.

It is well-known that in general the class of extending modules is not closed under direct sums, and this behavior is also carried on by Goldie extending modules and ADS-modules. Finding necessary and sufficient conditions for ensuring the closure of such classes under direct sums has been one of the most important open problems in the theory of extending modules and their generalizations. In the next parts of our work, we shall deal with such a problem for Goldie ADS modules. 
In order to obtain when a direct sum of two Goldie ADS modules has the same property, the following concept generalizing relative injectivity will be useful.

Lemma 3.5 ([3, Theorem 2.7]). Let $M_{1}$ and $M_{2}$ be modules such that $M=M_{1} \oplus M_{2}$. Then $M_{1}$ is $M_{2}$-ejective if and only if for every $K \leq M$ such that $K \cap M_{1}=0$, there exists $M_{3} \leq M$ such that $M=M_{1} \oplus M_{3}$ and $K \cap M_{3} \leq_{e} K$.

In [7, Lemma 3.1], it is shown that an $R$-module $M$ is ADS if and only if for each decomposition $M=A \oplus B, A$ and $B$ are mutually injective.

Lemma 3.6. An R-module $M$ is Goldie $A D S$ if and only if for each decomposition $M=A \oplus B, A$ and $B$ are mutually ejective.

Proof. Suppose $M=A \oplus B$ is Goldie ADS. We will show that $A$ is $B$-ejective. Let $K$ be a submodule of $M$ such that $K \cap A=0$. So $K$ is contained in a complement, say $C$, of $A$. Then, by hypothesis, there exists $D \leq M$ such that $C \beta D$ and $M=A \oplus D$. It is easy to see that $K \cap D \leq_{e} K$. Thus, we have $A$ is $B$-ejective by Lemma 3.5.

Conversely, suppose for each decomposition $M=A \oplus B, A$ and $B$ are mutually ejective. Let $C$ be a complement of $A$. By Lemma 3.5, there exists and $D \leq M$ such that $M=A \oplus D$ and $C \cap D \leq_{e} C$. So, $A \oplus(C \cap D) \leq^{e} M$. It follows that $C \cap D \leq_{e} D$. So we are done by Lemma 3.4 .

Let $M_{1}$ and $M_{2}$ be modules with $Z\left(M_{1}\right)=0$ and $M=M_{1} \oplus M_{2}$. In [3, Corollary 2.8], it is shown that $M_{1}$ is $M_{2}$-injective if and only if $M_{1}$ is $M_{2}$-ejective. As the authors pointed out in [3], if $Z(M)=0$ and $M$ is $R$-ejective, then $M$ is injective (because of the Baer criterion).

Corollary 3.7. A non-singular Goldie ADS module is ADS.

Proof. By Lemma 3.6 and [3, Corollary 2.8].

We collect, in the following theorem, some fundamental properties of Goldie ADS modules.

Theorem 3.8. Assume that $M$ is Goldie ADS. Then the following statements hold.

1. Every direct summand of $M$ is Goldie ADS.

2. $M$ satisfies (C3) condition on fully invariant summands.

3. For any decomposition $M=A \oplus B$ and any $b \in B, A$ is bR-ejective.

Proof. (1) Assume that $A$ is a direct summand of $M$, i.e., $M=A \oplus B$ for some $B \leq M$. Let $A=A_{1} \oplus A_{2}$ and $K$ be a complement of $A_{1}$ in $A$. Then we have $M=A_{1} \oplus\left(A_{2} \oplus B\right)$. First we show that $K \oplus B$ is a complement of $A_{1}$ in $M$. Let $C \leq M$ such that $K \oplus B \leq C$ and $C \cap A_{1}=0$. Then $K \leq C \cap A$ and $(C \cap A) \cap A_{1}=0$. Since $K$ is a complement of $A_{1}$ in $A$, we can obtain that $K=C \cap A$. It follows that

$$
K \oplus B=(C \cap A) \oplus B=C \cap(A \oplus B)=C .
$$

Since $M$ is Goldie ADS, there exists a submodule $D$ of $M$ such that $(K \oplus B) \beta D$ and $M=A_{1} \oplus D$. Hence $A=A_{1} \oplus(D \cap A)$. It is easy to see that $K \beta(D \cap A)$.

(2) Let $A$ and $B$ be fully invariant direct summands of $M$ such that $A \cap B=0$. We shall show that $A \oplus B$ is a direct summand of $M$. Write $M=A \oplus A^{\prime}$ and $M=B \oplus B^{\prime}$ 
for some submodules $A^{\prime}, B^{\prime}$ of $M$. By Lemma 3.6, $A$ is $A^{\prime}$-ejective. Hence there exists $M^{\prime} \leq M$ such that $M=M^{\prime} \oplus A$ and $B \cap M^{\prime} \leq_{e} B$ by Lemma 3.5. Inasmuch as $B$ is a fully invariant submodule of $M, B=\left(M^{\prime} \cap B\right) \oplus(A \cap B)=M^{\prime} \cap B$. It follows that $B \leq M^{\prime}$. Then $M^{\prime}=B \oplus\left(M^{\prime} \cap B^{\prime}\right)$ and so $M=A \oplus B \oplus\left(M^{\prime} \cap B^{\prime}\right)$.

(3) Suppose $M$ has a decomposition $M=A \oplus B$. By Lemma 3.6, the module $A$ is $B$-ejective. Let $K=A \oplus b R$ and $X$ be a submodule of $K$ such that $X \cap A=0$. Since $A$ is $B$-ejective, there exists $C \leq M$ such that $M=A \oplus C$ and $X \cap C \leq_{e} X$ by Lemma 3.5. Note that $K=A \oplus(C \cap K)$. It follows that $(C \cap K) \cap X=X \cap C \leq_{e} X$. Now $A$ is $b R$-ejective for any $b \in B$ by Lemma 3.5 .

A module $M_{R}$ is called Goldie quasi continuous if $M$ is Goldie extending and satisfies (C3) (see [3]).

Proposition 3.9. Every Goldie quasi continuous module is Goldie ADS.

Proof. Assume that $M$ has a decomposition $M=S \oplus T$ and $T^{\prime}$ is a complement of $S$ in $M$. Since $M$ is Goldie extending, there exists a direct summand $D$ of $M$ such that $T^{\prime} \beta D$. Since $T^{\prime} \cap S=0$ we have $D \cap S=0$ by the equivalence relation $\beta$. We have $S \oplus\left(T^{\prime} \cap D\right) \leq^{e} M$ and obtain that $S \oplus D \leq^{e} M$. So, by (C3) property, we obtain that $M=S \oplus D$.

In [11], Kuratomi defined the GQC (generalized quasi continuous) modules by using Goldie extending modules. $M$ is said to be $G Q C$ if for every submodule $X_{1}$ and $X_{2}$ of $M$ with $X_{1} \cap X_{2}=0$ there exists an essential submodules $Y_{i} \leq_{e} X_{i}$ and a decomposition $M=A_{1} \oplus A_{2}$ such that $Y_{i}$ is a submodule of $A_{i}$ for $i=1,2$. Let $\left\{M_{i}: i \in I\right\}$ be a family of modules. The direct sum decomposition $M=\oplus_{I} M_{i}$ is said to be exchangeable if, for any direct summand $X$ of $M$, there exists $\bar{M}_{i} \leq M_{i}(i \in I)$ such that $M=X \oplus\left(\oplus_{I} \bar{M}_{i}\right)$. A module $M$ is said to have the finite internal exchange property (FIEF) if, any finite direct sum decomposition $M=M_{1} \oplus M_{2} \oplus \cdots \oplus M_{n}$ is exchangeable.

Corollary 3.10. The following statements are equivalent for a duo module $M$ :

1. $M$ is Goldie extending and Goldie ADS.

2. $M$ is Goldie quasi continuous.

3. $M$ is GQC with FIEP.

Proof. By Theorem 3.8, Proposition 3.9 and [11, Theorem 3.4].

We have the following direct sum decomposition theorem for Goldie extending submodules.

Lemma 3.11 ([11, Proposition 2.1]). Let $M, N, M_{i}$ and $N_{i}$ be modules.

1. If $N$ is $M_{1}$-ejective and $M_{2}$-ejective, then $N$ is $M_{1} \oplus M_{2}$-ejective.

2. Let $M_{1}$ be a direct summand of $M$ and $N_{1}$ a direct summand of $N$. If $M$ is $N$-ejective, then $M_{1}$ is $N_{1}$-ejective.

3. If $M_{1}$ and $M_{2}$ are $N$-ejective modules then so is $M_{1} \oplus M_{2}$.

The proof of the following proposition uses the similar argument as in [14]. 
Proposition 3.12. Let $M=\bigoplus_{i=1}^{n} M_{i}$ be direct sum of fully invariant submodules $M_{i}$ 's . Then $M$ is Goldie $A D S$ if and only if each $M_{i}$ is Goldie $A D S$ and $M_{i}$ is $M_{j}$-ejective for all $i, j=1,2, \ldots, n$ and $i \neq j$.

Proof. $\Longrightarrow$ : By Theorem 3.8 (1), $M_{i}$ 's are Goldie ADS. Again by Theorem 3.8 (2), $M_{i} \oplus M_{j}$, which is a direct summand of $M$ for $i \neq j$, is Goldie ADS. But by Lemma 3.6, $M_{i}$ is $M_{j}$-ejective for $i \neq j$.

$\Longleftarrow$ : Let $M=A \oplus B$. We claim that $A$ is $B$-ejective. Since each $M_{i}$ is fully invariant, we can obtain that $M_{i}=\left(A \cap M_{i}\right) \oplus\left(B \cap M_{i}\right)$ for all $i=1,2, \ldots, n$ by [2]. It follows that $A=\bigoplus_{i=1}^{n}\left(A \cap M_{i}\right)$ and $B=\bigoplus_{i=1}^{n}\left(B \cap M_{i}\right)$. Since $M_{i}$ is Goldie ADS, $\left(A \cap M_{i}\right)$ is $\left(B \cap M_{i}\right)$-ejective. Since $M_{i}$ is $M_{j}$-ejective then $\left(A \cap M_{i}\right)$ is $\left(B \cap M_{j}\right)$-ejective for all $i, j=1,2, \ldots, n$ by Lemma $3.11(2)$. It follows that $A \cap M_{i}$ is $B$-ejective for all $i=1,2, \ldots, n$ by Lemma $3.11(1)$. Thus $A$ is $B$-ejective by Lemma $3.11(3)$.

$E(-)$ denotes the injective hull for a module.

Theorem 3.13. The following conditions are equivalent for a module $M$ :

1. $M$ is Goldie ADS.

2. For every decomposition $M=A \oplus B$, for all $f \in \operatorname{Hom}(E(B), E(A))$, there exists $D \leq M$ such that $M=A \oplus D$ and $D \beta X$, where $X=\{b+f(b) \mid b \in B, f(b) \in A\}$.

Proof. (1) $\Rightarrow(2)$ We show that $X=\{b+f(b) \mid b \in B, f(b) \in A\}$ is a complement of $A$ in $M$. First, we note that that $A \cap X=0$. Let $L$ be a submodule of $M$ such that $L \cap A=0$ and $X \leq L$. Consider the the natural projections $\pi_{A}$ and $\pi_{B}$ of $M$ onto $A$ and $B$, respectively.

Claim: $\pi_{A}(x)=f \pi_{B}(x)$ for all $x \in L$. Assume that there exists $x \in L$ such that $\left(\pi_{A}-\right.$ $\left.f \pi_{B}\right)(x) \neq 0$. Since $A \leq_{e} E(A)$, there exists $r \in R$ such that $0 \neq\left(\pi_{A}-f \pi_{B}\right)(x r) \in A$. But $\pi_{A}(x r)-f \pi_{B}(x r)=x r-\left(\pi_{B}(x r)+f \pi_{B}(x r)\right) \in A \cap L=0$, a contradiction. Thus $\pi_{A}(x)=f \pi_{B}(x)$ for all $x \in L$.

Now, let $x \in L$. Hence $x=a+b$, where $a \in A$ and $b \in B$. Then $\pi_{A}(x)=a$. By the claim, we can obtain $\pi_{A}(x)=a=f \pi_{B}(x)$ for all $x \in L$. Therefore, $x=a+b=$ $f \pi_{B}(x)+b \in X$. It follows that $L=X$. The rest is clear from the definition of Goldie $\mathrm{ADS}$.

(2) $\Rightarrow$ (1) Let $M=A \oplus B$, and $T$ be a complement of $A$ in $M$.

Then $T=\{k+f(k) \mid k \in K\}$ for some $K \leq B$ and $f \in H o m(E(B), E(A))$. In fact, let $\pi_{B}: A \oplus B \rightarrow B$ be the canonical projection. There exists $f: E(B) \rightarrow E(A)$ such that $f \pi_{B}(t)=t-\pi_{B}(t)$ for all $t \in T$. Thus $T=\left\{k+f(k) \mid k \in \pi_{B}(T)\right\}$. By (2), there exists $D \leq M$ such that $M=A \oplus D$ and $D \beta X$, where $X=\{b+f(b) \mid b \in B, f(b) \in A\}$. Note that $f\left(\pi_{B}(T)\right) \leq A$ and so $D \cap T \leq_{e} T$. Now we show that $T \cap D \leq_{e} D$. Let $d \in D, d \neq 0$. Assume $T \cap d R=0$. If $(T \oplus d R) \cap A \neq 0$, write $a=c+d r \neq 0$ for some $a \in A, c \in T$ and $r \in R$. We have $c \neq 0$ and obtain that there exists $r^{\prime} \in R$ such that $c r^{\prime} \in T \cap D$ and $c r^{\prime} \neq 0$. Therefore $a r^{\prime}=c r^{\prime}+d r r^{\prime} \in A \cap D=0$, which implies $c r^{\prime}+d r r^{\prime}=0$. It follows that $c r^{\prime}=-d r r^{\prime} \in T \cap d R=0$ hence $c r^{\prime}=0$, a contradiction. Thus $(T \oplus d R) \cap A=0$ and then $T=T \oplus d R$ by the maximality of 
$T$. It follows that $d=0$, again a contradiction. Thus we get $T \cap d R \neq 0$. Hence there exists $y \in R$ such that $d y \in T \cap D$ and $d y \neq 0$. So $D \cap T \leq_{e} D$.

\section{Goldie ADS rings}

A ring $R$ is called a right Goldie ADS ring if $R_{R}$ is a Goldie ADS module.

We start this section with the following ring extension.

Theorem 4.1. Let $M$ be a $S-R$-bimodule. Assume that

$$
T=\left(\begin{array}{cc}
S & M \\
0 & R
\end{array}\right)
$$

is right Goldie ADS. Then

1. $R$ is right Goldie $A D S$

2. $M_{R}$ is Goldie $A D S$.

Proof. (1) Let $R_{R}=A \oplus B, I \leq A$ and $f: I \rightarrow B$ an $R$-homomorphism. Let

$$
\bar{A}=\left(\begin{array}{ll}
0 & 0 \\
0 & A
\end{array}\right), \bar{B}=\left(\begin{array}{cc}
0 & 0 \\
0 & B
\end{array}\right) \text { and } \bar{I}=\left(\begin{array}{ll}
0 & 0 \\
0 & I
\end{array}\right) .
$$

It is easy to see that $\bar{A} \oplus \bar{B}$ is a direct summand of $T_{T}$. We define $\theta: \bar{I} \rightarrow \bar{B}$ via

$$
\theta\left(\left(\begin{array}{ll}
0 & 0 \\
0 & r
\end{array}\right)\right)=\left(\begin{array}{cc}
0 & 0 \\
0 & f(r)
\end{array}\right)
$$

Then $\theta$ is a $T$-homomorphism. By the hypothesis, there exists a $T$-homomorphism $\phi: \bar{A} \rightarrow \bar{B}$ and $\bar{J} \leq_{e} \bar{I}$ such that $\phi(\bar{j})=\theta(\bar{j})$ for every $\bar{j} \in \bar{J}$, where

$$
\bar{J}=\left(\begin{array}{ll}
0 & 0 \\
0 & J
\end{array}\right) .
$$

It is clear that $\phi$ is an $R$-homomorphism. Let $\iota: A \rightarrow \bar{A}$ via

$$
\iota(a)=\left(\begin{array}{ll}
0 & 0 \\
0 & a
\end{array}\right)
$$

and $\pi: \bar{B} \rightarrow B$ via

$$
\pi\left(\left(\begin{array}{ll}
0 & 0 \\
0 & b
\end{array}\right)\right)=b
$$

Then $\iota$ and $\pi$ are $R$-homomorphisms. Since $\bar{J} \leq_{e} \bar{I}$ then $J \leq_{e} I$. Let say $\bar{f}:=\pi \phi \iota$. So

$$
\bar{f}(j)=\pi \phi \iota(j)=\pi \phi\left(\left(\begin{array}{ll}
0 & 0 \\
0 & j
\end{array}\right)\right)=\pi \theta\left(\left(\begin{array}{ll}
0 & 0 \\
0 & j
\end{array}\right)\right)=\pi\left(\left(\begin{array}{cc}
0 & 0 \\
0 & f(j)
\end{array}\right)\right)=f(j)
$$

for every $j \in J$ so we are done by Lemma 3.6.

(2) Assume that that $M_{R}=M_{1} \oplus M_{2}, N \leq M_{1}$ and $f: N \rightarrow M_{2}$ is an $R$ homomorphism. Let

$$
\bar{M}_{1}=\left(\begin{array}{cc}
S & M_{1} \\
0 & 0
\end{array}\right), \bar{M}_{2}=\left(\begin{array}{cc}
0 & M_{2} \\
0 & R
\end{array}\right) \text { and } \bar{N}=\left(\begin{array}{cc}
0 & N \\
0 & 0
\end{array}\right) .
$$


It is easy to see that $T_{T}=\bar{M}_{1} \oplus \bar{M}_{2}$. We define $\theta: \bar{N} \rightarrow \bar{M}_{2}$ via

$$
\theta\left(\left(\begin{array}{ll}
0 & n \\
0 & 0
\end{array}\right)\right)=\left(\begin{array}{cc}
0 & f(n) \\
0 & 0
\end{array}\right) .
$$

Then $\theta$ is a $T$-homomorphism. By the hypothesis, there exists a $T$-homomorphism $\phi: \bar{M}_{1} \rightarrow \bar{M}_{2}$ and $\bar{J} \leq_{e} \bar{N}$ such that $\phi(\bar{j})=\theta(\bar{j})$ for all $\bar{j} \in \bar{J}$. Then $\phi$ is an $R$-homomorphism. Let $\iota: M_{1} \rightarrow \bar{M}_{1}$ via

$$
\iota(m)=\left(\begin{array}{cc}
0 & m \\
0 & 0
\end{array}\right)
$$

and $\pi: \bar{M}_{2} \rightarrow M_{2}$ via

$$
\pi\left(\left(\begin{array}{cc}
0 & m \\
0 & r
\end{array}\right)\right)=m .
$$

Then $\iota$ and $\pi$ are $R$-homomorphisms. Since $\bar{J} \leq_{e} \bar{N}$ then $J \leq_{e} N$. Say $\bar{f}:=\pi \phi \iota$ so $\bar{f}(j)=f(j)$.

We recall the following useful lemma proved in [5, Lemma 5].

Lemma 4.2. Let $M$ be a right $R$-module, and let $L$ be a submodule of $M$, where $R=R e R$ for some $e^{2}=e \in R$ and $S=e R e$. Then:

1. $L$ is essential in $M$ if and only if Le is essential in $(M e)_{S}$;

2. $L$ is a complement in $M$ if and only if Le is a complement in $(M e)_{S}$;

3. $L$ is a direct summand of $M$ if and only if Le is a direct summand of $(M e)_{S}$.

Proposition 4.3. Let $M$ be a right $R$-module, where $R=R e R$ for some $e^{2}=e \in R$ and $S=e R e$. Then:

1. $(M e)_{S}$ is a Goldie ADS module if and only if $M_{R}$ is a Goldie ADS module.

2. $(R e)_{S}$ is a Goldie $A D S$ if and only if $R_{R}$ is Goldie $A D S$.

Proof. (1) Assume that $(M e)_{S}$ is a Goldie ADS module. Let $M_{R}=X \oplus Y$ and $Z$ be a submodule of $M$ with $Z \cap X=0$. Then $M e=X e \oplus Y e$ and, by Lemma 4.2, $Z e \cap X e=0$. Since $(M e)_{S}$ is a Goldie ADS module, there exists a submodule $D$ of $M e$ such that $Z e \cap D$ is essential in $Z e$ and $M e=X e \oplus D$. Hence $M_{R}=X \oplus D R$ and $Z \cap D R$ is essential in $Z$. They imply that $X$ is $Y$-ejective by Lemma 3.5, and hence $M_{R}$ is a Goldie ADS module by Lemma 3.6.

Assume that $M_{R}$ is a Goldie ADS module. Let $M e=D \oplus T$ and $K$ be a submodule of $(M e)_{S}$ with $K \cap D=0$. Then $M=D R \oplus T R$ and, by Lemma 4.2, $K R \cap D R=0$. Since $M_{R}$ is a Goldie ADS module, there exists a submodule $X$ of $M$ such that $K R \cap X$ is essential in $K R$ and $M=D R \oplus X$. Hence $M e=(D R) e \oplus X S$ and $(K R) e \cap X e R \leq X S$. Now $D R e=D e R e=D$ and $K R e=K e R e=K$. This implies that $M e=D \oplus X S$ and $K \cap X S$ is essential in $K$. Thus $D$ is $T$-ejective by Lemma 3.5, and hence $(M e)_{S}$ is a Goldie ADS module by Lemma 3.6.

(2) It is a direct consequence of (1).

Theorem 4.4. $M_{n}(R)$ is Goldie $A D S$ if and only if $\left(\oplus_{i=1}^{n} R_{i}\right)_{R}$ is Goldie ADS, where each $R_{i}=R$. 
Proof. It is easy to obtain that $M_{n}(R)=M_{n}(R) e M_{n}(R)$, where $e$ is the matrix unit with 1 in the $(1,1)$ th position and zero elsewhere. The rest is follows by Proposition 4.3 .

\section{References}

[1] Abdioglu, C., Zemlicka, J., On type-ADS modules and rings, Journal of Algebra and Its Applications, https://doi.org/10.1142/S0219498818500834.

[2] Agayev, N., Leghwel, A., Koşan, M.T., Harmanci, A., Duo modules and duo rings, Far East J. Math. Sci, 20(2005), no. 3, 341-346.

[3] Akalan, E., Birkenmeier, G.F, Tercan, A., Goldie extending modules, Commun. Algebra, 37(2009), no. 3, 663-683.

[4] Akalan, E., Birkenmeier, G.F., Tercan, A., Corrigendum to: Goldie extending modules, Commun. Algebra, 38(2010), 4747-4748.

[5] Akalan, E., Birkenmeier, G.F., Tercan, A., Goldie extending rings, Comm. Algebra, 40(2012), no. 3, 423-428.

[6] Akalan, E., Birkenmeier, G.F., Tercan, A., Corrigendum to: Goldie extending modules, Commun. Algebra, 41(2013), no. 4, 2005-2005.

[7] Alahmadi, A., Jain, S.K., Leroy, A., ADS modules, J. Algebra, 352(2012), 215-222.

[8] Amin, I., Yousif, M., Zeyada, N., Soc-Injective Rings and Modules, Comm. Algebra, 33(11)(2005), 4229-4250.

[9] Dung, N.V., Huynh, D.V., Smith, P.F., Wisbauer, R., Extending modules, Pitman Research Notes in Math. Longman, 1994, 313.

[10] Fuchs, L., Infinite Abelian Groups, vol. I, Pure Appl. Math. Ser. Monogr. Textb. vol. 36, Academic Press, New York, San Francisco, London 1970.

[11] Kuratomi, Y., Goldie extending modules and generalizations of quasi-continuous modules, Tsukuba J. Math, 38(2014), no. 1, 25-37.

[12] Mohammed, S.H., Müller, B.J., Continous and Discrete Modules, London Math. Soc. LN. Cambridge Univ. Press, 1990, 147.

[13] Nicholson, W.K., Yousif, M.F., Quasi-Frobenius Rings, Cambridge Univ. Press, 2003.

[14] Quynh, T.C., Koşan, M.T., On ADS modules, Commun. Algebra, 42(2014), 3541-3551.

Truong Cong Quynh

Department of Mathematics, Danang University

459 Ton Duc Thang, DaNang city, Vietnam

e-mail: tcquynh@dce.udn.vn

Serap Şahinkaya

Department of Mathematics, Faculty of Science

Gebze Technical University

Kocaeli, Turkey

e-mail: srpsahinkaya@gmail.com 